\title{
Exotic Chemistry with Ultracold Rydberg Atoms
}

\author{
Heiner Saßmannshausen§, Johannes Deiglmayr, and Frédéric Merkt \\ §SCS-DSM Award for best poster presentation in Physical Chemistry
}

\begin{abstract}
We review recent experiments carried out with dense $\left(10^{12} \mathrm{~cm}^{-3}\right)$ ultracold $(T=40 \mu \mathrm{K})$ samples of Cs atoms which have the goal to characterize, by high-resolution spectroscopy, the interactions between Cs atoms, $\mathrm{Cs}^{+}$ions and electrons that lead to the formation of metastable long-range molecules. The types of molecules observed in these experiments and the mechanisms leading to the aggregation of atoms in weakly bound molecules are very different from those encountered in warmer samples. In particular, we present results on molecules with binding energies of less than $0.05 \mathrm{~J} / \mathrm{mol}$ and discuss their properties in the context of a new category of molecular states arising from slow-electron-atom scattering and their relation to atomic and molecular Rydberg states. One of the astonishing aspects of these types of molecules is that they can still be treated in good approximation in the realm of the Born-Oppenheimer approximation despite a huge electronic-state density. Non-Born-Oppenheimer effects are revealed by the decay of the molecules into neutral and charged fragments.
\end{abstract}

Keywords: Dipole-dipole interactions · Long-range Rydberg molecules · Rydberg states .

Singlet and triplet electron-Cs scattering length - Ultracold Rydberg gases

\section{Rydberg States and Long-range Rydberg Molecules}

Powerful methods to cool down atomic samples to ultracold temperatures, ${ }^{[1-4]}$ i.e. temperatures below $1 \mathrm{mK}$, offer chemists the opportunity to study atom/atom interactions and molecule formation in a regime previously not accessible. This regime is characterized, in our experiments, by a typical temperature of $40 \mu \mathrm{K}$ corresponding to a thermal energy of $h \cdot 800 \mathrm{kHz}$ or $0.3 \mathrm{~mJ} /$ mol and to an extremely low average velocity of $0.05 \mathrm{~m} / \mathrm{s}$ in the case of Cs atoms. Such conditions enable the investigation of fragile molecules bound by much less than the thermal energy at room temperature. Such molecules can be formed and studied by high-resolution photoassociation spectroscopy starting from ultracold atomic samples. Using ultracold samples in spectroscopic experiments has the additional advantages of negligible Doppler widths $(200 \mathrm{kHz}$ in the UV and for Cs atoms at $40 \mu \mathrm{K})$ and long interaction times, resulting in small energy uncertainties, relevant,

\footnotetext{
${ }^{*}$ Correspondence: Prof. Dr. F. Merkt

Laboratory of Physical Chemistry

ETH Zurich

Vladimir-Prelog-Weg 2

$\mathrm{CH}-8093$ Zürich

E-mail: feme@xuv.phys.chem.ethz.ch
}

e.g. for the spectroscopic determination of binding energies. These advantages are currently exploited in an increasing range of applications in atomic and molecular physics and in chemistry focusing on the fundamental understanding of interatomic and intramolecular long-range interactions (see e.g. refs $[5,6]$ ) and precision measurements of atomic and molecular properties (see e.g. refs [7-10]).

Rydberg states of atoms and molecules are at the heart of the research presented in this contribution. These states consist of a positively charged ion core and an excited electron (the Rydberg electron) located in a large orbit of dimension given approximately by the Bohr radius $a_{0} n^{2}$, where $n$ is the principal quantum number. At sufficiently high values of $n$, the Rydberg electron is insensitive to the structure of the positively charged ion core, and the Rydberg level structure becomes similar to that of the hydrogen atom. High Rydberg states of all atoms and molecules can be described in good approximation by Rydberg's empirical formula ${ }^{[11,12]}$

$$
E(n)=E_{I}\left(\alpha^{+}\right)-\frac{h c R_{M}}{\left(n-\delta_{l}\right)^{2}},
$$

where $h, c, E_{\mathrm{I}}\left(\alpha^{+}\right), R_{\mathrm{M}}$, and $\delta_{l}$ are Planck's constant, the speed of light in vacuum, the energy of the ionic state $\alpha^{+}$relative to the

Table 1. Scaling of selected properties of atoms in high Rydberg states of principal quantum number $n$. Numerical values correspond to the $33 p_{3 / 2}$ state of Cs.

\begin{tabular}{|l|cc|}
\hline Property & $\boldsymbol{n}$ scaling & Cs $\left(\mathbf{3 3 p}_{\mathbf{3} / 2}\right)$ \\
\hline Binding energy & $n^{-2}$ & $127 \mathrm{~cm}^{-1}, 16 \mathrm{meV}, 3.8 \mathrm{THz}$ \\
\hline Orbital radius & $n^{2}$ & $70 \mathrm{~nm}$ \\
\hline Radiative lifetime & $n^{3}$ & $>50 \mu \mathrm{s}$ \\
\hline Induced dipole moment & $n^{2}$ & 3000 Debye \\
\hline Local electron density & $n^{-6}$ \\
\hline Polarizability & $n^{7}$ & \\
\hline $\mathrm{C}_{6}$ van der Waals coefficient & $n^{11}$ & \\
\hline
\end{tabular}


neutral ground state, the mass-dependent Rydberg constant and the quantum defect, which, in any non-hydrogenic atom or molecule, depends on the orbital angular momentum quantum number $l$ and deviates from zero in penetrating low- $l$ states.

The scaling with $n$ of the physical properties of Rydberg atoms and molecules most relevant to our studies are summarized in Table 1. This Table illustrates that high Rydberg states have a large orbital radius, are weakly bound, long lived, highly polarisable, and easily perturbed by ground-state or Rydberg atoms in their vicinity. The physical properties of atoms or molecules in high Rydberg states are primarily determined by their quantum state and are thus universal.

The experiments summarized below rely on the combination of ultracold samples of Cs atoms prepared in a magnetooptical trap (MOT) and the unusual properties of Rydberg states at high $n$ values. These experiments have been described in more detail in refs [10,13-16].

\section{Experimental}

The experiments were performed under ultrahigh vacuum using ultracold samples of Cs atoms released from a MOT at a density of $10^{12}$ atoms $/ \mathrm{cm}^{3}$ and a temperature of $40 \mu \mathrm{K}$. All atoms were prepared in either the $F=3$ or the $F=4$ hyperfine component of the $6 s_{1 / 2}$ ground state, from which they were photoexcited to high $n \mathrm{p}_{3 / 2}$ Rydberg states in single-photon transitions in the ultraviolet (UV) at a wavelength of $\sim 319 \mathrm{~nm}$. The UV radiation driving the transitions is either obtained by doubling the frequency of the continuous-wave output of a singlemode ring dye laser at $\sim 639 \mathrm{~nm}$ resulting in a bandwidth of $1 \mathrm{MHz}$ in the UV, or by pulse amplification of the $639 \mathrm{~nm}$ radiation in dye cells, followed by frequency doubling in a BBO crystal, resulting in $5 \mathrm{~ns}$ long UV pulses with a bandwidth of 140 $\mathrm{MHz}$ at a repetition rate of $10 \mathrm{~Hz}$. The frequency of the laser is calibrated with a wavemeter and with a frequency comb. ${ }^{[10]}$ The Rydberg atoms or molecules are detected by applying pulsed electric potentials to a pair of electrodes surrounding the photoexciation region, causing pulsed field ionisation and extraction of the ions towards a charged-particle detector through an ion time-of-flight mass spectrometer. The resulting $\mathrm{Cs}^{+}$and $\mathrm{Cs}^{2+}$ ions are detected in separate channels, which enables us to detect molecular products resulting from the interaction of Cs Rydberg atoms with other Cs atoms located in their vicinity. Before photoexcitation, we turn off all optical and magnetic trapping fields and carefully compensate remaining electric and magnetic stray fields.

\section{Precision Measurements of Rydberg States of Cs}

When we carry out precision measurements of atomic properties we i) operate the trap at low atom densities to avoid broadening by interactions of the Rydberg atoms with ground-state atoms, ii) use low laser intensities to avoid AC Stark shifts of the transitions, and, more importantly, to avoid van-der-Waals interactions between Rydberg atoms, and iii) minimize the temperature of the trapped sample to reduce Doppler broadening. Under these conditions, we can record transitions from the $6 s_{1 / 2}$ ground state to $n p$ Rydberg states of $\mathrm{Cs}$ with a resolution (full width at half maximum) of $1 \mathrm{MHz}$ as illustrated in Fig. 1a. When the laser frequency is calibrated with a frequency comb, the line centers can be determined at an absolute accuracy of $60 \mathrm{kHz}$. Extrapolation of the measured Rydberg series using Rydberg's formula (Eqn. (1)), taking into account the energy dependence of the quantum defect up to the term linear in energy, enabled us to determine the first ionization energy of Cs $\left(E_{\mathrm{I}}=h c \cdot 31406.4677325(14) \mathrm{cm}^{-1}\right)$ to extreme precision and to extract improved values of the quantum defects of the p Rydberg series and their energy dependence.. ${ }^{[10]}$ Such a high precision and accuracy can only be achieved by careful compensation of stray magnetic and electric fields. We accomplish this by monitoring and minimizing the frequency shifts of the microwave transition between the $F=3$ and $F=$ 4 hyperfine components of the $6 s_{1 / 2}$ ground state and of the $n \mathrm{p}_{3 / 2} \leftarrow 6 \mathrm{~s}_{1 / 2}$ transitions using solenoids and electrodes designed for this purpose. An exemplary measurement of the stray electric field along one spatial coordinate is presented in Fig. 1b. The measured $70 \mathrm{p}_{3 / 2} \leftarrow 6 \mathrm{~s}_{1 / 2}$ transition is shifted almost exclusively by the quadratic Stark effect of the $70 \mathrm{p}_{3 / 2}$ Rydberg state. The externally applied electric-field strengths are given on the $y$ axis of Fig. 1b. At the point of maximal frequency (i.e. minimal quadratic Stark shift) the stray electric field in the direction of the externally applied field is compensated. ${ }^{[17]}$ This point lies at the apex of the parabola presented as a dashed black line. Measuring the Stark effect of high Rydberg states by applying electric fields in all three spatial dimensions enables us to reduce a background electric stray field of $\sim 0.3 \mathrm{~V} / \mathrm{cm}$ to below $1 \mathrm{mV} / \mathrm{cm}$. This level of compensation is necessary to avoid asymmetric line broadenings and shifts in precision measurements of Rydberg transitions and to achieve the afore-mentioned precision and accuracy. A full account of this investigation is given in ref. [10].

\section{Pressure Shift, Fermi Pseudo- potential and Long-range Rydberg Molecules}

At the maximal atom density in our MOT, the average distance between neighbouring atoms is on the order of $1 \mu \mathrm{m}$ and the probability that ground-state atoms are located within the large Rydberg orbit (diameter of about $140 \mathrm{~nm}$ at $n=33$, see Table 1) becomes significant. We can therefore study interactions between Rydberg and ground-state atoms in detail by observing how the Rydberg spectrum is affected by the increased atom density, ultimately leading to the well-known pressure shift of Rydberg transitions. This shift was first measured by Amaldi and Segré in 1934[18] and interpreted by Fermi ${ }^{[19]}$ as arising from the scattering of the Rydberg electron with atoms located within the electron orbit.
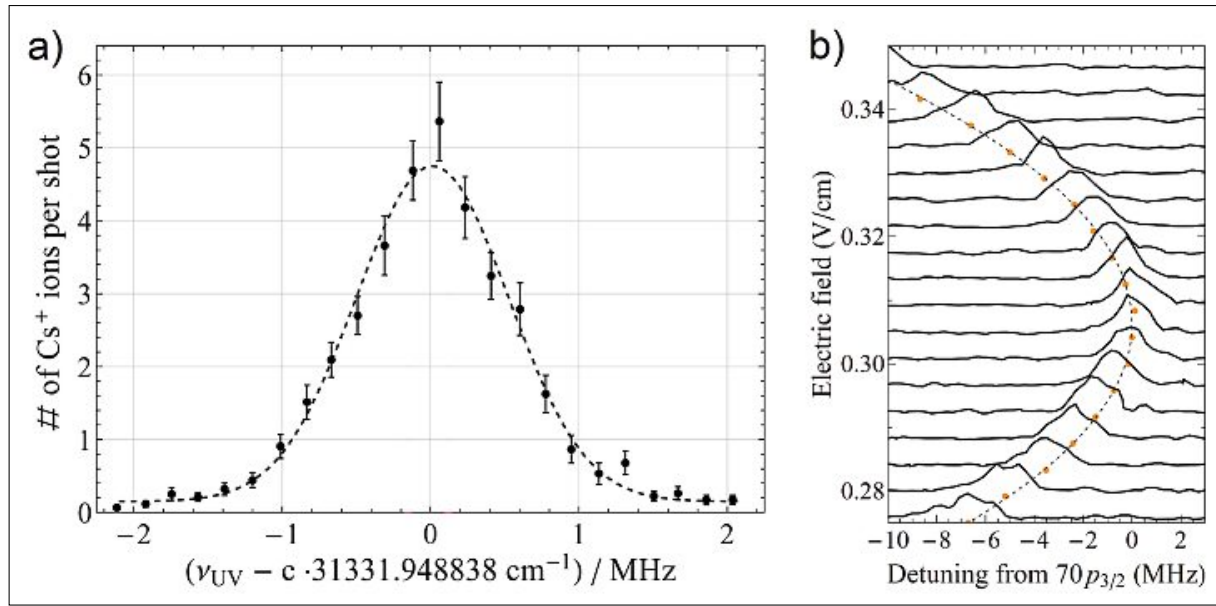

Fig. 1. a) Example of a high-resolution recording of the $42 p_{1 / 2} \leftarrow 6 s_{1 / 2}$ transition. b) Example for the spectroscopic determination of the electric stray field along the lab-frame $z$ axis. The figure contains several spectra of the $70 p_{3 / 2} \leftarrow 6 s_{1 / 2}$ transition measured in various externally applied electric fields. The values of the applied electric-field strengths correspond to the vertical offset of the spectra. The line centers are marked by orange dots and the dashed line indicates the parabolic curve fitted to the observed quadratic Stark shifts. 
This scattering can be treated using what is now known as a Fermi-contact-type pseudo-potential

$$
V(R)=2 \pi A_{0}|\Psi(R)|^{2}
$$

In this model, the interaction between the Rydberg and the ground-state atoms is reduced to the 'local' interaction between the electron, with the electron density at the position $R$ of the ground-state atom relative to the Rydberg ion core given by the square of the Rydberg electron wavefunction $|\Psi(R)|^{2}$. In Eqn. (2), $A_{0}$ is the zero-energy s-wave scattering length of the electron-atom collision. Because the Rydberg electron has a very small kinetic energy at the distance of high probability around the classical outer turning point, the model considers s-wave scattering only, and all higher partial waves do not contribute because the energy of the collision is assumed to be lower than the centrifugal barrier in the electron-atom scattering for $l>0$. Fermi showed that Eqn. (2) adequately describes the pressure shifts and broadenings of Rydberg lines arising from the presence of many ground-state atoms inside the Rydberg-electron orbit.[19]

In the limit of a single ground-state atom within the Rydberg-electron orbit and for a negative scattering length $A_{0}$, the Fermi pseudo-potential leads to the existence of a shallow oscillating molecular potential $V(R)$, proportional to the Rydberg electron density. ${ }^{[20]}$ Greene and coworkers ${ }^{[21]}$ pointed out that $V(R)$ can actually bind the Rydberg atom to the ground-state atom, giving rise to long-range Rydberg molecules with rovibrational levels that could be observed by high-resolution spectroscopy in ultracold gases. The size of these long-range Rydberg molecules is given by the size of the Rydberg-electron orbit and is enormous, about $50 \mathrm{~nm}$ at $n=30$. The existence of these long-range molecules was confirmed experimentally in studies of $n \mathrm{~s}_{1 / 2}$ ( $n=32-36$ ) Rydberg states of $\mathrm{Rb}$ in an ultracold sample by Pfau and coworkers.[22] The molecular potential can be refined by considering a linear energy dependence of the scattering length on the electron quasiclassical momentum $k^{[23]}$

$$
A(k)=A_{0}+\frac{\pi}{3} \alpha k(R),
$$

where $\alpha$ is the polarisability of the groundstate atom. The kinetic energy $k^{2} / 2$ of the Rydberg electron corresponds to the difference between the total energy and the Coulomb potential energy using the relation (in atomic units)

$$
\frac{1}{2} k^{2}=-\frac{1}{2 n^{2}}+\frac{1}{R}
$$

In this model, the only parameter needed to describe the molecular states is the zero-energy electron-atom scattering length $A_{0}$, for which calculations exist for many different elements. The calculated values of $A_{0}$ for the rare-gas atoms and the alkali-metal atoms are presented in Fig. 2 as a function of the polarisability of the atom in its ground state. In the case of the rare-gas atoms with completely filled subshells, only one scattering channel exists. The alkali-metal atoms with a single valence electron in the $n$ s atomic orbit ( $n$ $=2,3,4,5$, and 6 for $\mathrm{Li}, \mathrm{Na}, \mathrm{K}, \mathrm{Rb}$, and Cs, respectively) exhibit a singlet $(S=0)$ and a triplet $(S=1)$ scattering channel depending on the relative orientation of the spins of the valence electron and the scattered (Rydberg) electron. Fig. 2 reveals a surprisingly simple, linear scaling of the scattering lengths with the polarisabilities $\alpha$ of the ground-state atoms. It also reveals range molecules resulting from triplet scattering than for singlet scattering channels. This difference can be attributed to the exchange interaction between the Rydberg electron and the valence electron of the ground-state atom.

High-resolution photoassociation spectroscopy of long-range Rydberg molecules and a fit of their binding energies on the basis of Eqn. (2) have yielded the first a stronger binding interaction in the long- experimental determination of the singlet scattering length of electron-Cs collisions, ${ }^{[13]}$ and later, following the same model, also for electron-Rb collisions. [29] The experimental observations confirmed the calculations and also the linear scaling of $A_{0}$ with $\alpha$. Our contribution to the studies of these rather unusual molecular states came from the realization that the hyperfine interaction in the ground state of Cs (and of the lighter alkali-metal atoms) is larger than the interaction described by Eqn. (2) for the Rydberg states with $n$ in the range $25-40$ studied experimentally (see discussion in refs $[13,30]$ ). The atom thus couples the electron spin to its own nuclear spin, which effectively mixes singlet and triplet channels and gives rise to a second class of long-range molecular character and even weaker molecular binding energies than the first class of purely triplet scattering character.[13] An example for the resulting potential-energy curves and vibrational eigenstates is given in Fig. 3a for $33 p_{3 / 2}$.

In the Rydberg spectra, the molecular states appear on the low-frequency side of the atomic resonances, see Fig. $3 b$ for a measurement in the vicinity of the $33 \mathrm{p}_{3 / 2}$ $\leftarrow 6 \mathrm{~s}_{1 / 2}$ transition. The spectrum reveals among several other lines two strong lines. The first one, marked with a thick blue assignment bar at the top of Fig. 3b, appears at larger detuning from the atomic transition and arises from pure triplet hyperfine interaction of the ground-state states with mixed singlet-triplet scattering

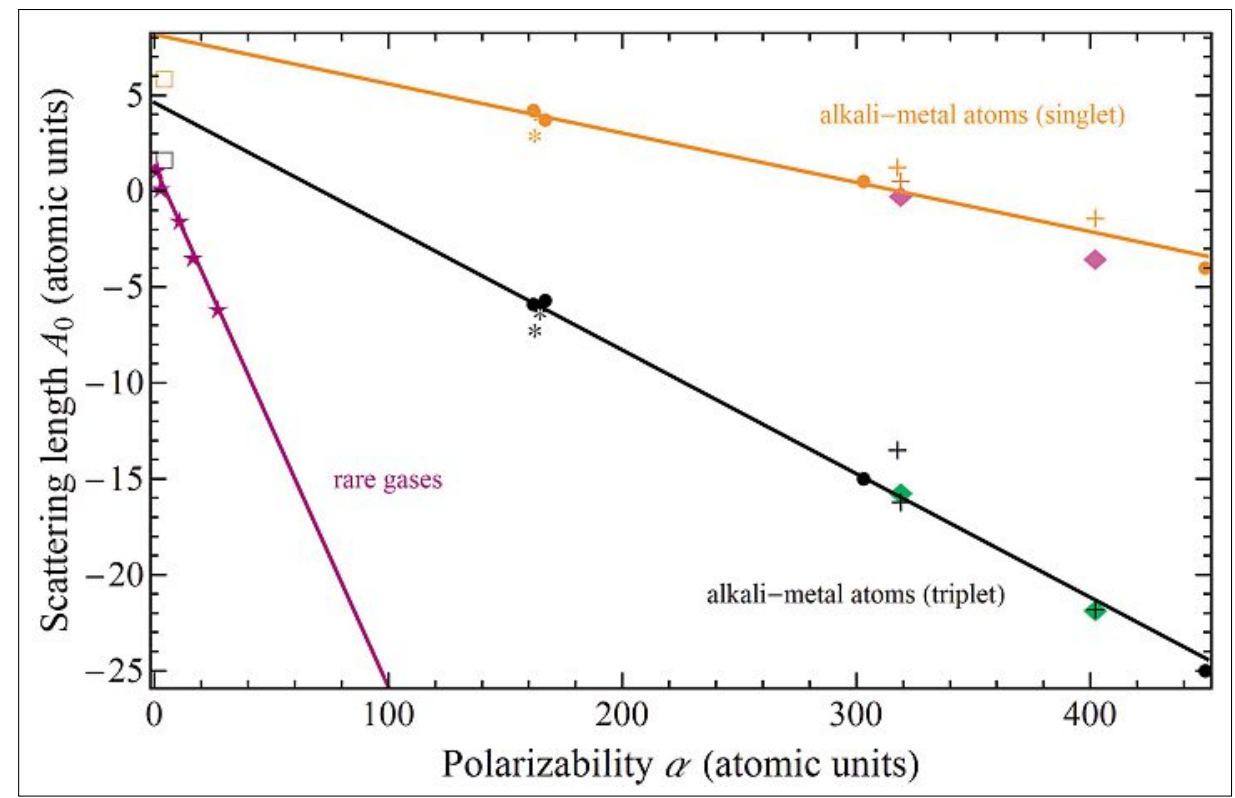

Fig. 2. Electron-atom scattering lengths $A_{0}$ as a function of the polarisability $\alpha$ for different elements and scattering channels. Values for the rare gases $\mathrm{He}, \mathrm{Ne}, \mathrm{Ar}, \mathrm{Kr}$ and $\mathrm{Xe}$ are taken from ref [24] and are indicated by purple five-pointed stars. For the alkali-metal atoms $\mathrm{Li}, \mathrm{Na}, \mathrm{K}, \mathrm{Rb}$ and Cs, the singlet and triplet scattering lengths are presented in orange and black, respectively (data taken from refs [25-27]). The singlet and triplet electron- $\mathrm{H}$ scattering lengths (data taken from ref [28]) are indicated by open squares. The values determined from a fit to high-resolution longrange Rydberg photoassociation spectroscopy are shown in green (triplet) and magenta (singlet)

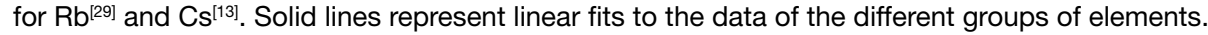



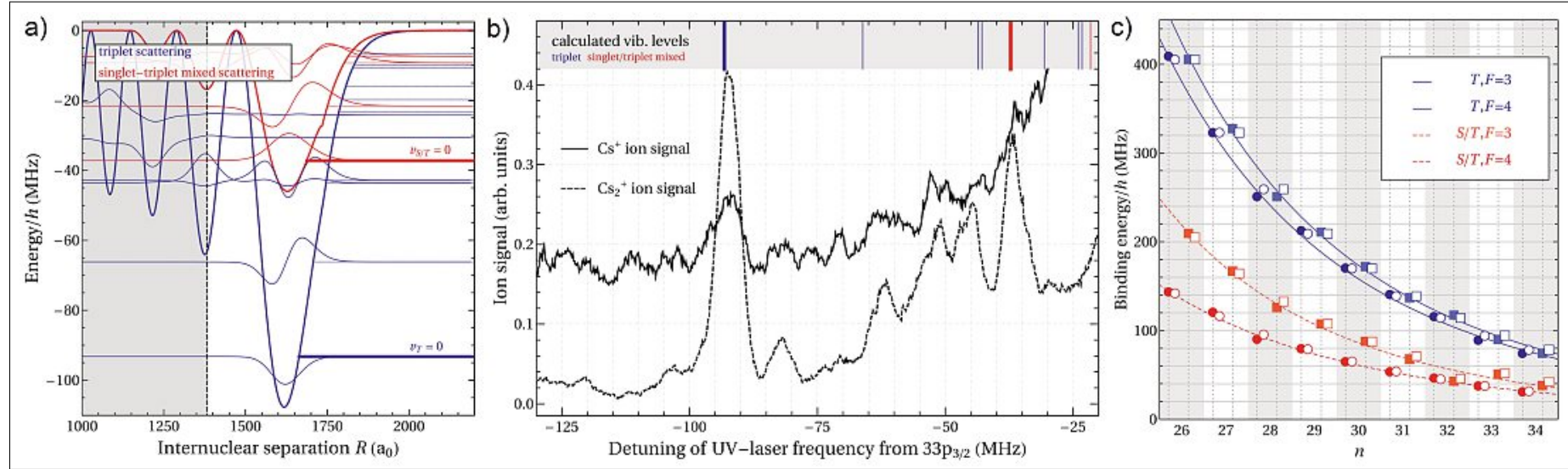

Fig. 3. a) Born-Oppenheimer (BO) potential energy curves calculated for the long-range $\mathrm{Cs}_{2}$ Rydberg molecular states resulting from pure triplet scattering (blue line) and mixed singlet-triplet scattering located near the $\mathrm{Cs} 6 \mathrm{~s}_{1 / 2}(F=4)+\mathrm{Cs}_{33 p_{3 / 2}}$ dissociation asymptote. The wavefunctions of the vibrational states are indicated by thin, the binding energies of the vibrational ground states by thick lines. b) Experimental spectra recorded on the low-frequency side of the atomic $33 \mathrm{p}_{3 / 2} \leftarrow 6 \mathrm{~s}_{1 / 2}(F=4)$ transition. The $\mathrm{Cs}^{+}$and $\mathrm{Cs}_{2}{ }^{+}$ion signals are displayed as full and dashed lines, respectively. The spectra were measured with a $0.5 \mu$ s long $150 \mathrm{~mW}$ UV pulse, and a delay of $5 \mu$ s between photoexcitation and pulsed-field ionisation. The spectral positions of vibrational levels calculated on the basis of the BO potentials displayed on the left-hand side of the figure are indicated by vertical lines at the top of the panel. c) Comparison of the binding energies (solid symbols) of all measured long-range molecules in their vibrational ground states in the range of principal quantum numbers between 26 and 34 with the calculated values (open symbols).

scattering. The second one, marked with a thick red assignment bar, is observed at smaller detuning and results from mixed singlet-triplet scattering. At the positions of the molecular resonances, we actually photoassociate two ultracold Cs atoms and form a long-range metastable $\mathrm{Cs}_{2}$ molecule. When we detect the molecules by pulsed field ionisation, we predominantly observe $\mathrm{Cs}_{2}{ }^{+}$ions (see Fig. 3b). The calculated positions of the vibrational ground states agree well with our experimental results over a wide range of $n$ values ( $n=$ 26-34), as illustrated by Fig. 3c.

For a chemist it may seem extraordinary that potential functions of the type represented in Fig. 3a can at all faithfully describe molecules in an energy region where the electronic-state density sis enormous and the Born-Oppenheimer approximation would be expected to fail. The reason why this is not the case is that, although the electronic motion is slow, the nuclear motion is still much slower at the low temperature of the atom sample and the large distances where photoassociation into these molecules takes place. A full account of this investigation is given in ref. [13].

\section{Rydberg-atom Dimers Interacting Through the van der Waals Interaction}

A final example illustrating the unusual properties of Rydberg atoms and their interactions is presented in Fig. 4, which shows the formation of molecular resonances resulting from the interaction between two Cs Rydberg atoms. Because of the scaling of the van der Waals coefficients with $n^{11}$ (see Table 1), the long-range interactions between two ultracold atoms in high- $n$ Rydberg states can dominate the dynamics of the system. To treat the long-range van der Waals interaction theoretically, one usually expresses it as a multipole expansion, the leading and often dominating term being the dipole-dipole interaction, which results formally in configuration interactions between near-degenerate levels of the atom pair, such as
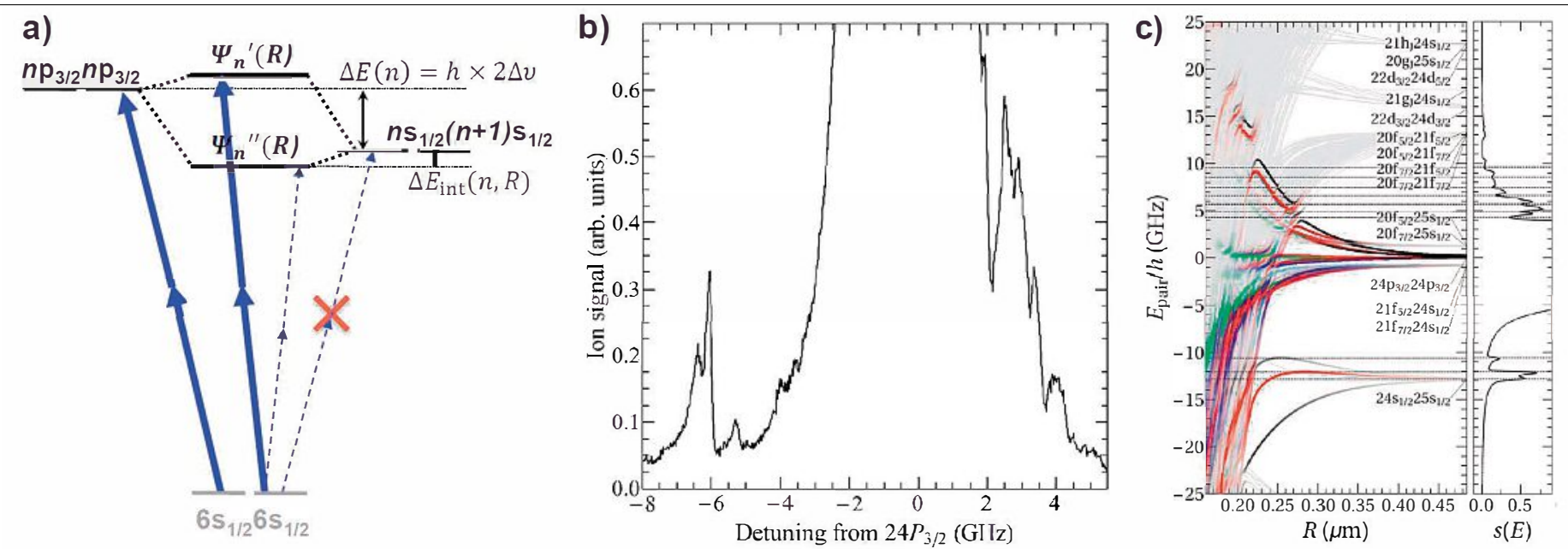

Fig. 4. a) Simplified and schematic configuration-interaction diagram describing the interactions between two Rydberg atoms at long range. Laser excitation is symbolized by blue arrows. The excitation of non-interacting atoms is only allowed to $n p$ states (thick arrows), and forbidden, e.g., to $n \mathrm{~s}_{1 / 2}$ states (dashed thin arrows). $\Psi(R)$ are the molecular states resulting from the long-range interactions. b) Spectrum recorded in the vicinity of the $24 p_{3 / 2} \leftarrow 6 s_{1 / 2}$ transition with a 5-ns-long pulsed UV laser. c) Calculated potential-energy curves of interacting Rydberg-atom-pair states. The colour coding refers to the $n p$ character of the molecular states. The spectrum displayed on the right-hand side was simulated on the basis of the potential-energy functions, as explained in detail in ref [16]. 


$$
\begin{aligned}
& n \mathrm{p}_{3 / 2}+n \mathrm{p}_{3 / 2} \stackrel{\text { dipole-dipole coupling }}{\longleftrightarrow} \\
& n \mathrm{~s}_{1 / 2}+(n+1) \mathrm{s}_{1 / 2} .
\end{aligned}
$$

This interaction is schematically depicted in the simplified energy-level diagram of Fig. 4a. The long-range interaction between the two Rydberg atoms leads to an $R$-dependent configuration mixing and to 'bonding' $\Psi_{n}^{\prime \prime}$ and 'anti-bonding' $\Psi_{n}^{\prime}$ molecular eigenstates. These two molecular eigenstates $\Psi(\mathrm{R})$ have mixed $n \mathrm{p}_{3 / 2} n \mathrm{p}_{3 / 2}$ and $n \mathrm{~s}_{1 / 2}(n+1) \mathrm{s}_{1 / 2}$ character and are shifted in energy by $\Delta E_{\text {int }}$ compared to the pairdissociation asymptotes constituted by the sum of the two Rydberg levels of the noninteracting atoms.

The dipole-dipole interaction mixes the optically accessible p Rydberg states with Rydberg states of $s$ character that are not accessible in single-photon transitions from the $6 \mathrm{~s}$ ground state. The intensity of the transitions to the mixed molecular states, formed by the interacting Rydberg atoms, is proportional to the $\mathrm{p}$-character of the mixed states.

The observation of such resonances $^{[6,31-34]}$ requires the simultaneous excitation of two Cs atoms to interacting Rydberg states. With a single monochromatic laser this corresponds to a two-photon excitation. The laser frequency at the position of the two-photon resonances is detuned from the $n \mathrm{p}_{3 / 2} \leftarrow 6 \mathrm{~s}_{1 / 2}$ resonances of the isolated atoms (see Fig. 4a). Because the strength of the two-photon transition rapidly decreases with increasing detuning from the atomic transition, we observe these molecular Rydberg-atom pairs only when the ultracold sample of $\mathrm{Cs}$ is illuminated with very intense UV laser pulses.

A typical spectrum recorded with a $5 \mathrm{~ns}$ long pulsed, intense $\left(\sim 10 \mathrm{MW} / \mathrm{cm}^{2}\right)$ UV laser is presented in Fig. 4b. The laser frequency is calibrated with a wavemeter and is referenced to the atomic $24 \mathrm{p}_{3 / 2} \leftarrow$ $6 s_{1 / 2}$ transition, which is strongly saturated under the conditions where the Rydbergatom-pair states are observed. Next to the saturated atomic resonance, we observe the density-dependent appearance of additional, sharp ( $\sim 300 \mathrm{MHz}$ FWHM) resonances at negative and positive detunings from the atomic resonance. To assign the molecular resonances observed experimentally it is necessary to consider several interacting Rydberg-atom-pair states and to carefully model the $R$-dependent interactions. To this end, we set up a Hamiltonian matrix describing the relevant long-range interactions, primarily dipole-dipole interactions, with weaker contributions from dipolequadrupole ${ }^{[15]}$ and quadrupole-quadrupole ${ }^{[16]}$ interactions. Determining the eigenvalues of this interaction matrix as a function of the internuclear distance leads to sets of potential-energy functions, as illustrated in Fig. 4c for the spectral region near the $24 \mathrm{p}_{3 / 2} 24 \mathrm{p}_{3 / 2}$ dissociation asymptote.

Some spectral positions correspond closely to the energy of pair-dissociation asymptotes, e.g. the resonance at a detuning of $\sim-6 \mathrm{GHz}$ in the spectrum depicted in Fig. 4b, which results from molecular states correlated at long range to the $23 \mathrm{~s}_{1 / 2} 24 \mathrm{~s}_{1 / 2}$ asymptote. The resonances on the high-frequency side result from quadrupole-quadrupole interactions between $n \mathrm{p}_{3 / 2} n \mathrm{p}_{3 / 2}$ and $n \mathrm{f} n$ 'f Rydberg-atom pair states. ${ }^{[16]}$

The shift of the line observed at a detuning of $-6 \mathrm{GHz}$ from the position of the $23 \mathrm{~s}_{1 / 2} 24 \mathrm{~s}_{1 / 2}$ dissociation asymptote corresponds to the interaction-induced shift $\Delta E_{\text {int }}$, introduced in Fig. 4a. The resonance exhibits an asymmetric broadening and a red-degraded line shape. The line shape and the magnitude and sign of the interaction-induced shift can be almost perfectly modeled by our numerical calculations based on the potential curves presented in Fig. 4c, taking into account the fact that it is the p-character of the final state that gives rise to observable intensities. A full account of these studies is given in ref. [16].

\section{Conclusion}

In this short overview of our research on Cs Rydberg atoms in ultracold samples we have studied unusual electronically excited states of $\mathrm{Cs}_{2}$ with extremely weak binding energies. Because in these states the nuclear motion is still much slower than the slow electronic motion, the treatment of the energy-level structure based on the Born-Oppenheimer approximation remains approximately valid. The states we have observed include long-range Rydberg molecules bound by a Fermi-contact-type interaction of the electron with the Cs neutral atoms. The binding is weaker than the hyperfine interaction in the ground state of Cs, which results in two classes of states, one of which we have observed for the first time. The second type of molecules consists of two atoms both excited to high Rydberg states and interacting at long range via the dipole-dipole and higher order multipole-multipole interactions. The very large internuclear distances and the extremely weak bonds observed in these molecules make them fascinating objects of scientific investigations.

Received: December 30, 2015

[1] T. Hänsch. A. Schawlow, Opt. Commun. 1975, 13,68 .

[2] D. J. Wineland, W. M. Itano, Phys. Rev. A 1979, 20, 1521.

[3] E. L. Raab, M. Prentiss, A. Cable, S. Chu, D. E. Pritchard, Phys. Rev. Lett. 1987, 59, 2631.

[4] C. Monroe, W. Swan, H. Robinson, C. Wieman, Phys. Rev. Lett. 1990, 65, 1571.

[5] K. M. Jones, E. Tiesinga, P. D. Lett, P. S. Julienne, Rev. Mod. Phys. 2006, 78, 483.

[6] S. M. Farooqi, D. Tong, S. Krishnan, J. Stanojevic, Y. P. Zhang, J. R. Ensher, A. S. Estrin, C. Boisseau, R. Côté, E. E. Eyler, P. L. Gould, Phys. Rev. Lett. 2003, 91, 183002.

[7] A. Marte, T. Volz, J. Schuster, S. Dürr, G. Rempe, E. G. M. van Kempen, B. J. Verhaar, Phys. Rev. Lett. 2002, 89, 283202.

[8] L. Carr, J. Ye, New J. Phys. 2009, 11, 055009.

[9] M. Mack, F. Karlewski, H. Hattermann, S. Höckh, F. Jessen, D. Cano, J. Fortágh, Phys. Rev. A 2011, 83, 052515.

[10] J. Deiglmayr, H. Herburger, H. Saßmannshausen, P. Jansen, H. Schmutz, F. Merkt, Phys. Rev. A 2016, 93, 013424.

[11] J. R. Rydberg, Z. Phys. Chem. 1890, 5, 227.

[12] T. F. Gallagher, 'Rydberg Atoms', Cambridge University Press, Cambridge, 1994.

[13] H. Saßmannshausen, F. Merkt, J. Deiglmayr, Phys. Rev. Lett. 2015, 114, 133201 .

[14] H. Saßmannshausen, F. Merkt, J. Deiglmayr, Phys. Rev. A 2013, 87, 032519.

[15] J. Deiglmayr, H. Saßmannshausen, P. Pillet, F. Merkt, Phys. Rev. Lett. 2014, 113, 193001.

[16] H. Saßmannshausen, F. Merkt, J. Deiglmayr, Phys. Rev. A 2015, 92, 032505 .

[17] A. Osterwalder, F. Merkt, Phys. Rev. Lett. 1999, 82, 1831.

[18] E. Amaldi, E. Segrè, Il Nuovo Cimento 1934, $11,145$.

[19] E. Fermi, Il Nuovo Cimento 1934, 11, 157.

[20] V. Kokoouline, O. Dulieu, R. Kosloff, F. Masnou-Seeuws, J. Chem. Phys. 1999, 110, 9865.

[21] C. H. Greene, A. S. Dickinson, H. R. Sadeghpour, Phys. Rev. Lett. 2000, 85, 2458.

[22] V. Bendkowsky, B. Butscher, J Nipper, J. P. Shaffer, R. Löw, T. Pfau, Nature 2009, 458, 1005.

[23] A. Omont, J. Phys. (Paris) 1977, 38, 1343.

[24] K. Rupnik, U. Asaf, S.P. McGlynn, J. Chem. Phys. 1990, 92, 2303.

[25] E. Karule, Phys. Lett. 1965, 15, 137.

[26] D. W. Norcross, J. Phys. B: At. Mol. Phys. 1971, 4,1458

[27] C. Bahrim, U. Thumm, I. I. Fabrikant, J. Phys. B: At. Mol. Opt. Phys. 2001, 34, L195.

[28] C. Schwartz, Phys. Rev. 1961, 124, 1468.

[29] F. Böttcher, A. Gaj, K. M. Westphal, M. Schlagmüller, K. S. Kleinbach, R. Löw, T. Cubel Liebisch, T. Pfau, S. Hofferberth, arXiv:1510.01097, 2015.

[30] D. A. Anderson, S. A. Miller, G. Raithel, Phys. Rev. A 2014, 90, 062518.

[31] J. Stanojevic, R. Côté, D. Tong, S. M. Farooqi, E. E. Eyler, P. L. Gould, Eur. Phys. J. D 2006, $40,3$.

[32] J. Stanojevic, R. Côté, D. Tong, E. E. Eyler, P. L. Gould, Phys. Rev. A, 2008, 78, 052709.

[33] K. R. Overstreet, A. Schwettmann, J. Tallant, J. P. Shaffer, Phys. Rev. A 2007, 76, 011403.

[34] K. R. Overstreet, A. Schwettmann, J. Tallant, D. Booth, J. P. Shaffer, Nat. Phys. 2009, 5, 581 . 